\title{
Instructional Animations: More Complex to Learn from Than at First Sight?
}

\author{
Anna Wong ${ }^{1}$, Nadine Marcus ${ }^{1}$, and John Sweller ${ }^{2}$ \\ ${ }^{1}$ School of Computer Science and Engineering, \\ University of New South Wales, Sydney, Australia \\ \{annawong, nadinem\} @cse.unsw. edu.au \\ ${ }^{2}$ School of Education, \\ University of New South Wales, Sydney, Australia \\ j.sweller@unsw.edu.au
}

\begin{abstract}
This paper presents a cognitively guided set of design guidelines for instructional animations based on a review of the existing literature. The guidelines are based around a cognitive load theory framework, which assumes learners' limited working memories must be considered for instructional animations to be effective. We propose six design guidelines: (1) animations are more beneficial for learners with higher levels of prior knowledge; (2) animations are more effective for certain knowledge domains; (3) segment animations in shorter sections; (4) give learners control; (5) signal or cue important information; (6) remove details and information from instructional animations that are not necessary for learning.
\end{abstract}

Keywords: Cognitive load theory, animation, multimedia instructional design.

\section{Introduction}

Instructional animations are commonly used in many different types of user interfaces, particularly in computer-based educational environments. Additionally, the increasingly common use of new UI technologies such as interactive whiteboards and multi-touch devices, combined with increasing affordability of high-bandwidth Internet and the popularity of video streaming sites, make animations increasingly accessible in the classroom. Despite the appeal of animations, the factors influencing their effectiveness are not fully understood, often making appropriate implementation in educational settings difficult.

Instructional animations are a series of still frames that, when considered together, are perceived as motion, used for educational purposes. Existing research in animation has produced mixed results. Many studies show no intrinsic superiority of animation over static graphics [1], unless other design techniques to support learning were also introduced, such as learner control [2] or segmentation into shorter sections [3]. Other research shows animation to be highly effective for learning, but only when depicting human movement tasks [4]. The range of results indicates the effectiveness of animations is heavily influenced by a number of factors, making design guidelines even more critical to designers and educators alike. 
Instructional animations are often difficult to learn from because they are transient [5]. Information appears on the screen and then disappears in a very short period of time, making it very difficult for information to be reviewed. Humans possess a working memory that can only store and process small amounts of information very briefly [6]. Our working memory limitations combined with the transience of animations mean that novel, complex information presented in an animated format can be challenging for learners to process. Transience inherent in most instructional animations means learners need to simultaneously remember and process both previously presented information as well as currently presented information to understand the learning material. However, previously learned information may have already been lost from working memory before the current information has been processed. In contrast, static graphics can be revisited on demand in a way that is more difficult to replicate using animations. The ability to revisit information in static graphics means previously presented information does not need to be held in working memory, thus reducing or potentially even eliminating transience and making materials easier to understand.

Although other design guidelines for instructional animations do exist, the pace at which technology evolves means they quickly become outdated (e.g., [7]), or are highly theoretical (e.g., [8]). Recent cognitive load related research can explain when and how animations can be effective.

\subsection{Cognitive Load Theory}

Cognitive load theory (CLT) [9] is a framework of research-based instructional design principles based on the characteristics and relationships between the structures within our human cognitive architecture, which persist regardless of age.

CLT is based around the notion of a working memory used to process current information, that is very limited in terms of both the quantity of novel information it can process and the duration such information can be stored [6]. In contrast, long-term memory is effectively infinite in terms of the quantity and duration it can store knowledge. Long-term memory is able to store such large amounts of information efficiently by organizing them into schemas, cognitive structures that help organize information according to how it will be used. Schemas brought in to working memory from long-term memory are treated as a single item in working memory, hence reducing working memory load. Central to CLT is the concept of cognitive load [9], the amount of mental effort exerted during learning or performing a task. There are three major types of cognitive load:

Intrinsic cognitive load is the cognitive load inherent in the learning materials. Intrinsic cognitive load cannot be altered without changing the meaningfulness of the content. It is determined primarily by the number of elements of information that needs to be considered simultaneously in working memory.

Extraneous cognitive load is the cognitive load arising from instructional design factors. This form of cognitive load can be changed through effective instructional design. Most classical cognitive load theory research has been concerned with lowering extraneous load.

Germane cognitive load is the load used to create schemas and automate them. 
CLT has been used successfully over the last 20 years to guide instructional designers. More recently, it has been successfully used to inform multimodal interface design [10]. The guidelines generated by CLT can help inform both design and use of instructional animations.

\section{Design and Teaching Implications}

As mentioned previously, a primary characteristic of animation is its transience, which can overload learners' limited working memory. As such, design techniques associated with animations primarily aim to decrease transience. The CLT research to date suggests the following design and teaching recommendations:

Instructional animations are better for learners with more prior knowledge. None of the design principles below can be considered in isolation of learners' level of prior knowledge. Prior knowledge stored in long-term memory in the form of schemas, determines how we make sense of information. Design of instructional materials must account for learners' prior knowledge. Animations are more beneficial for learners who already have some prior knowledge in an area [11]. Prior knowledge reduces transience, hence reducing or even eliminating working memory limitations because limitations only occur when dealing with novel information. Low prior knowledge learners benefit less from animation, as they do not have the schemas to attenuate transient information in animations.

Instructional animations are more useful for some knowledge domains over others. There is emerging evidence that animations depicting some specific domains, such as human movement, are more efficiently taught using animations [4]. This may be because animations depicting motor skills tap into our innate ability to learn through observing other people, thus reducing the load on working memory.

Segment instructional animations into shorter sections. Segmenting animations into smaller sections results in better learning compared to non-segmented animations, especially if the segments are combined with simple forms of learner control, for example letting learners move between sections at will [2]. Segmenting animations reduces the amount of transient information to be processed by working memory.

Give learners some control over the animation. Simple forms of learner control, such as pacing, can improve the effectiveness of animations [2]. However, it should not be relied upon in the absence of appropriate instructional design, as students may not use it appropriately, or at all [5]. Learner control should also be designed to accommodate prior knowledge - learners with low prior knowledge in the knowledge domain to be learnt and the software environment may benefit more by having access to simpler forms of learner control until they develop expertise, when more complex forms of learner control can be introduced.

Signal or cue to focus attention on what's important. Signaling or cueing important parts of an animation can help focus the learner's attention on the critical parts [3], decreasing working memory load imposed by visual search. However, cues need to be designed carefully to not distract learners' attention from the information to be learnt. 
Remove details and information from instructional animations that are not necessary for learning. Removing unnecessary information and details decreases extraneous cognitive load, making it easier for learners to focus attention where it is required [12]. Content and details chosen for inclusion in instructional animations should not distract from the focus of the learning task. Learners with high prior knowledge may be able to handle more detailed animations than novice learners.

\section{Conclusions}

Although new technologies show immense promise, it is important to understand learners have limited working memories. Instructional animations must be used and designed with these limitations in mind. The animation related guidelines listed are by no means complete and the use of animations still an active area of research. Rapid changes in technology mean that these guidelines will also continue to evolve. These guidelines also do not negate the need for appropriate instructional design of other learning materials. Nonetheless, we believe CLT and these guidelines can provide a useful learner-centered framework to help guide designers and educators alike.

\section{References}

1. Tversky, B., Morrison, J.B., Betrancourt, M.: Animation: can it facilitate? Int. J. HumComput. St. 57, 247-262 (2002)

2. Mayer, R.E., Chandler, P.: When learning is just a click away: Does simple user interaction foster deeper understanding of multimedia messages? J. Educ. Psychol. 93, 390-397 (2001)

3. Moreno, R.: Optimising learning from animations by minimizing cognitive load: Cognitive and affective consequences of signaling and segmentation methods. Appl. Cognitive Psych. 21, 765-781 (2007)

4. Wong, A., Marcus, N., Ayres, P., Smith, L., Cooper, G.A., Paas, F., Sweller, J.: Instructional animations can be superior to statics when learning human motor skills. Comput. Hum. Behav. 25, 339-347 (2009)

5. Hasler, B.S., Kersten, B., Sweller, J.: Learner control, cognitive load and instructional animation. Appl. Cognitive Psych. 21, 713-729 (2007)

6. Cowan, N.: The magical number 4 in short-term memory: A reconsideration of mental storage capacity. Behav. Brain Sci. 24, 87-185 (2000)

7. Nielsen, J.: Guidelines for multimedia on the web, http://www. useit.com/alertbox/9512.html

8. Ayres, P., Paas, F.: Can the cognitive load approach make instructional animations more effective? Appl. Cognitive Psych. 21, 811-820 (2007)

9. Sweller, J.: Instructional Design in Technical Areas. ACER Press, Camberwell (1999)

10. Oviatt, S.: Human-centered design meets cognitive load theory: Designing interfaces that help people think. In: Proceeding of the 16th ACM International Conference on Multimedia, pp. 871-880. ACM Press, New York (2006)

11. Kalyuga, S.: Relative effectiveness of animated and static diagrams: An effect of learner prior knowledge. Comput. Hum. Behav. 24, 852-861 (2008)

12. Mayer, R.E., Heiser, J., Lonn, S.: Cognitive constraints on multimedia learning: When presenting more material results in less understanding. J. Educ. Psychol. 93, 187-198 (2001) 